\title{
BEMERKUNGEN ZUR FUNKTION DER PALÄSTREN IN DEN EPHESISCHEN BAD-GYMNASIUM-KOMPLEXEN*
}

Vom Ende des 1. Jahrhunderts bis in die Mitte des 2. Jahrhunderts n. Chr. werden im Stadtgebiet von Ephesos vier Bad-Gymnasium-Komplexe errichtet (Hafen-, Theater-, Ost- und Vediusgymnasium $^{1}$ ), die grundrißtypologisch dem Idealtypus des seit dem Ende des 1. Jahrhunderts n. Chr. in Kleinasien nachweisbaren Thermengymnasiums entsprechen (Abb. 1). Bei drei dieser Anlagen (Theater-, Ost- und Vediusgymnasium) dominiert der Badblock gegenüber den angeschlossenen Palästren so augenscheinlich, daß Überlegungen zur Funktion dieser Peristylhöfe angestellt werden müssen².

Die architektonische Form der Palästra - ein quadratischer oder rechteckiger, von Säulenhallen umgebener, offener Platz - stammt aus Griechenland, wo sie in klassischer und hellenistischer Zeit sowohl eigenständig als auch als Teil des Gymnasions anzutreffen ist. Daß die Formulierung einer Unterscheidung der Begriffe 'Palästra' und 'Gymnasion' in ihrer antiken Verwendung ein Desiderat darstellt, wurde zuletzt von Ch. Wacker betont ${ }^{3}$. Grundsätzlich kann aber festgehalten werden, daß unter dem Überbegriff 'Gymnasion' - abgeleitet vom griechischen $\gamma \cup \mu \nu \alpha ́ \zeta \varepsilon ı v$ (nackt sein, trainieren) - verschiedene architektonische Einheiten, wie Dromos, Xystos, Stoa, Palästra und Grünanlagen, als integrierte Elemente subsumiert wurden ${ }^{4}$. Die Primärverwendung des Begriffes 'Gymnasion' in der Antike bezog sich folglich nicht auf ein

* Der Artikel entstand im Rahmen der wiederaufgenommenen Forschungstätigkeit am Vediusgymnasium in Ephesos. - Mein Dank gilt dem Leiter der Ausgrabungen in Ephesos, Herrn F. Krinzinger, für die materielle und intellektuelle Unterstützung dieser Arbeiten. Für zahlreiche inhaltliche Hinweise danke ich vor allem Herrn I. Weiler sowie Herrn G. A. Plattner, Herrn P. Scherrer, Frau R. Hanslmayr und Herrn H. Taeuber. - Abkürzungen und Zitierweise folgen den Richtlinien des Österreichischen Archäologischen Instituts; vgl. ÖJh 69, 2000, 357 ff. (http:// www.oeai.at/publik/autoren.html). Zusätzlich zu den dort angeführten wird hier folgende Abkürzung verwendet: IvE = Inschriften griechischer Städte aus Kleinasien 11, 1 (1979) - 17, 4 (1984), Ephesos (IvE Ia-VIII 2).

Es handelt sich dabei jeweils um moderne Bezeichnungen.

2 Allgemein zu Palästren: W. Zschietzschmann, Wettkampf- und Übungsstätten in Griechenland. Stadion - Palästra - Gymnasion. Eine Übersicht II (1961) bes. 31 f.; S. L. Glass, Palaistra and Gymnasium in Greek Architecture (1967) bes. 69 ff.; ders. in: W. J. Raschke (Hrsg.), The Archaeology of the Olympics. The Olympics and Other Festivals in Antiquity (1988) 155-173; D. G. Kyle, Athletics in Ancient Athens, 95. Suppl. Mnemosyne (1987) 64 ff.; E. Brödner, Die römischen Thermen und das antike Badewesen. Eine kulturhistorische Betrachtung (1983) 75 ff.; D. Krencker, Die Trierer Kaiserthermen. Abteilung I. Ausgrabungsbericht und grundsätzliche Untersuchungen römischer Thermen, Trierer Grabungen und Forschungen I 1 (1929) 186; I. Nielsen, Thermae et Balnea. The Architecture and Cultural History of Roman Public Baths² (1993) 163 f.; M. Weber, Antike Badekultur (1996) 40. Zur griechischen Palästra: J. Delorme, Gymnasion. Étude sur les monuments consacrés à l'éducation en Grèce, BEFAR 196 (1960) bes. 253 ff. Als Teil des kleinasiatischen Bad-Gymnasium-Komplexes: F. Yegül, Baths and Bathing in Classical Antiquity (1992) bes. $306 \mathrm{ff}$.

3 Ch. Wacker, Das Gymnasion in Olympia. Geschichte und Funktion, Würzburger Forschungen zur Altertumskunde 2 (1996) 13 f. - Erschwert wird die Zuordnung der Begriffe durch den Umstand, daß bereits in den antiken Quellen 'Gymnasion' und 'Palästra' unterschiedlich und z. T. mißverständlich verwendet werden; s. dazu Kyle (Anm. 2) 66 .

4 Vgl. auch die einschlägige Beschreibung bei Vitr. 5, 11; zur Vitruv-Stelle ausführlich: Glass (Anm. 2:1967) 77 f.; Brödner (Anm. 2) 76 f. - Allgemein zur Terminologie des Badewesens: R. Rebuffat in: Les thermes romains. Actes de la table ronde organisée par l'École française de Rome 1988, Collection de l'École française de Rome 142 (1991) 1 ff. 


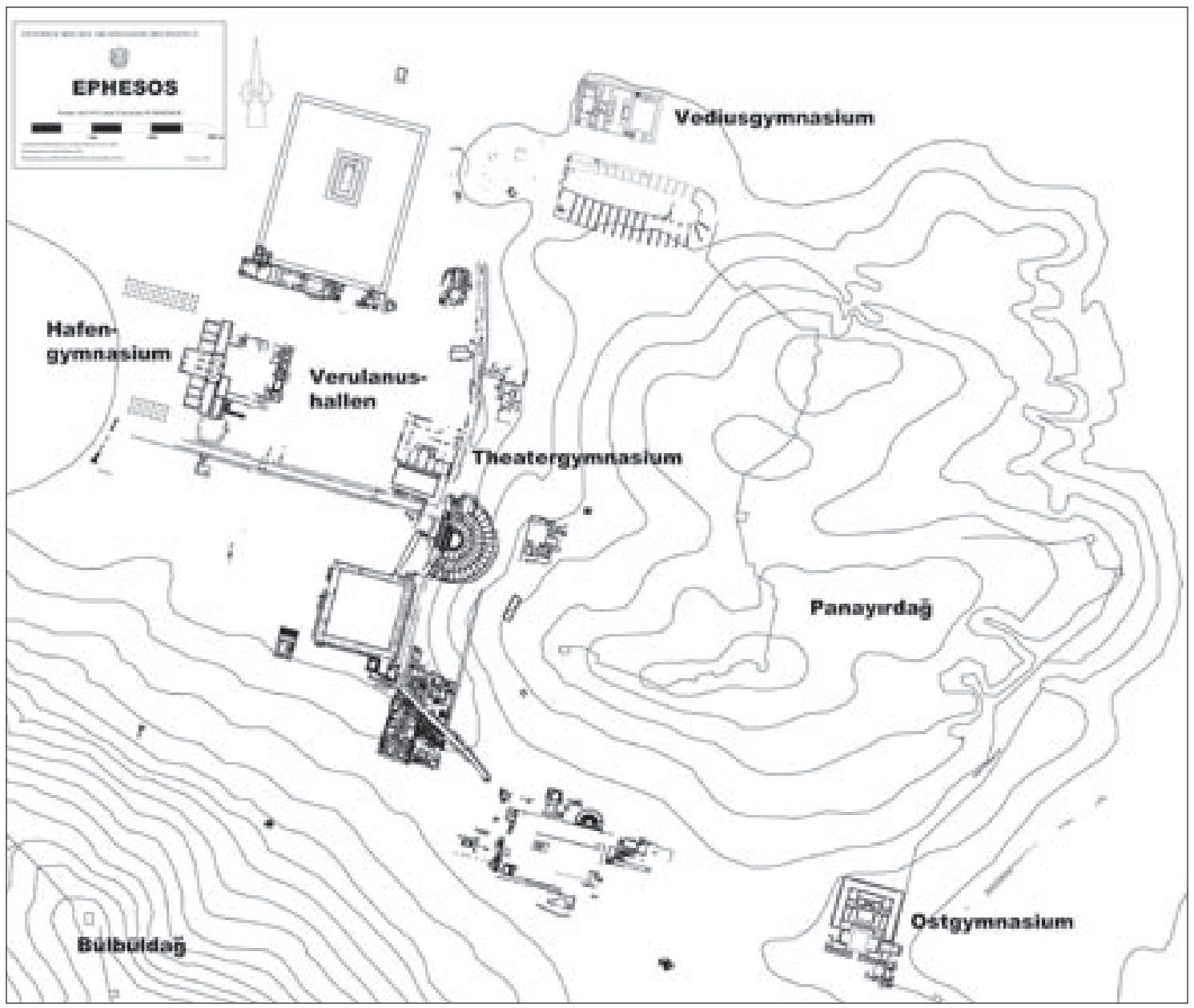

1 Ephesos. Stadtplan

Bauwerk, der Begriff bezeichnete vielmehr eine heterogene Gruppe unterschiedlichster architektonischer Formen. Die Palästra bildet dabei einen Bestandteil des Gymnasions ${ }^{5}$.

Der Begriff 'Palästra' wird etymologisch von $\pi \alpha \lambda \alpha i$ cıv (ringen) abgeleitet, beschreibt also ursprünglich einen Ringkampfplatz ${ }^{6}$. Bereits in den Beschreibungen von Platon ${ }^{7}$ tritt aber der philosophisch-musische, d. h. der intellektuelle Aspekt in der Benützung der Palästren immer mehr in den Vordergrund. Zur Zeit Ciceros ${ }^{8}$ scheinen die Gymnasien und in der Folge die Palästren schon weitgehend von Intellektuellen vereinnahmt gewesen zu sein 9 .

5 Wacker (Anm. 3) 13; so auch Zschietzschmann (Anm. 2) 36. - Zum Begriff 'Gymnasion’: Isid. etym. 15, 2, 30: Gymnasium generalis est exercitiorum locus.; Isid. etym. 15, 2, 41: Haec et gymnasia dicuntur, quia ibi athletae uncto corpore et perfricato manibus exercitantur; nam yvuváoiov Graece, Latine exercitium dicitur.

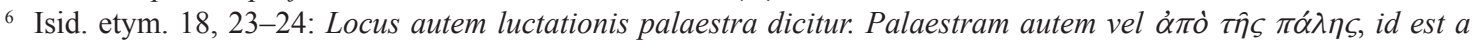
luctatione, ...

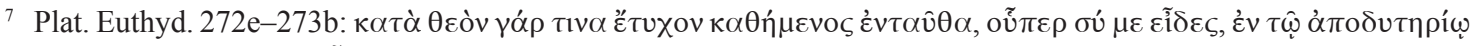

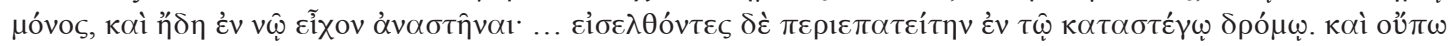

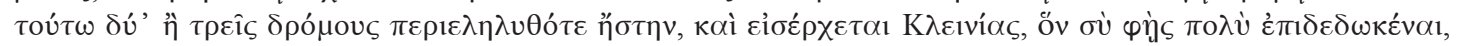

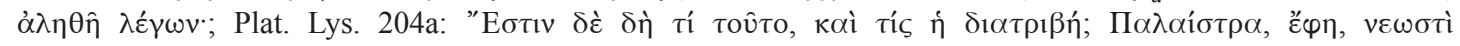

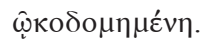

8 Cic. de or. 2, 5, 21: Omnia ista, inquit Crassus, ego alio modo interpretor, qui primum palaestram et sedis et porticus etiam ipsos, Catule, Graecos exercitationis et delectationis, inuenisse arbitror. Nam et saeculis multis ante gymnasia inuenta sunt quam in eis philosophi teneant, tamen eorum auditores discum audire quam philosophum malunt; ...

9 s. auch N. K. Rauh, BCH 116, 1992, 298. Vgl. in diesem Zusammenhang Ch. Roueché, Performers and Partisans at Aphrodisias in the Roman and Late Roman Periods, JRS Monographs 6 (1993) 135: »The original purpose of 
Am Ende des 1. Jahrhunderts n. Chr. entsteht in Ephesos ein neuer Typus des Gymnasiums, der die römische Therme (grundrißtypologisch jeweils Varianten des 'Kaisertypus' ${ }^{10}$ ) mit dem griechischen Gymnasion verbindet und modern meist als Bad-Gymnasium-Komplex umschrieben wird ${ }^{11}$. Elementare Bestandteile bilden der Badblock und die angeschlossene Palästra. Zumindest für zwei der vier ephesischen Anlagen kann ihre antike Bezeichnung rekonstruiert werden: Das heute als Vediusgymnasium bekannte Gebäude nördlich des Stadions wurde gemäß

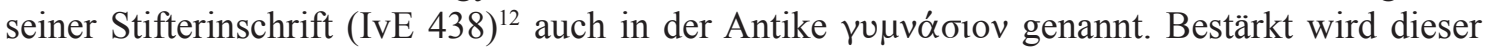
Umstand durch den Rhetor Aristeides, der seine Waschungen, die ihm von Asklepios aufgetragen

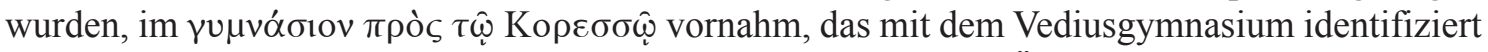
werden kann ${ }^{13}$. Wieder bildet die Bezeichnung 'Gymnasion' den Überbegriff für eine aus unterschiedlichen architektonischen Formen - Therme und Palästra - bestehende Gesamtanlage. Für das Hafengymnasium ist sowohl die Bezeichnung 'Kaiserthermen' ( $\beta \alpha \lambda \alpha v \varepsilon \hat{\alpha} \alpha \tau \hat{\omega} v \Sigma \varepsilon \varepsilon \beta \alpha \sigma \tau \hat{\omega} v$ bzw. $\Sigma \varepsilon \beta \alpha \sigma \tau o \hat{)})$ als auch 'Kaisergymnasium' ( $\Sigma \varepsilon \beta \alpha \sigma \tau o ̀ v ~ \gamma v \mu v \alpha ́ \sigma o v)$ überliefert ${ }^{14}$.

Welche Aussagen können nun über die Funktion der ephesischen Palästren getroffen werden ${ }^{15}$ ?

the Greek gymnasium was to train the youth of the cities for military service; even in its earliest form this training was associated with contests. As the need for such training diminished, it was replaced by the need to train the upper classes of the cities to excel in physical sports and also intellectually, in music, philosophy, rhetoric, and the other arts.«

10 Entsprechend der maßgeblichen Typologie bei Krencker (Anm. 2) $174 \mathrm{ff}$.

11 So z. B. von Yegül (Anm. 2) 250 ff.; F. K. Yegül, The Bath-Gymnasium Complex at Sardis, Sardis Reports 3 (1986); ders., ArtB 64, 1982, 7 ff.; A. Farrington in: S. Macready - F. H. Thompson (Hrsg.), Roman Architecture in the Greek World (1987) 50-59. - Nielsen (Anm. 2) 105 ff. spricht von »bath-gymnasia«, Brödner (Anm. 2) 75 ff. von »Thermengymnasien«. R. Maccanico, ArchCl 15, 1963, 32-60 verwendet »ginnasi« und F. Fasolo, L'architettura Romana di Efeso, Bollettino del centro di studi per la storia dell'architettura 18 (1962) 31-40 nicht ganz zutreffend »terme «.

12 IvE 438 (heute im Domitiansdepot/Ephesos - Fragmente einer marmornen Wandverkleidungsplatte, die einen Pfeiler zwischen dem Doppeltor von IIIa nach VI verblendete):

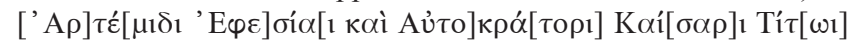

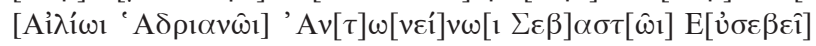

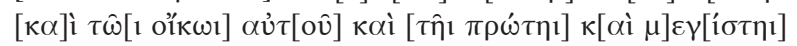

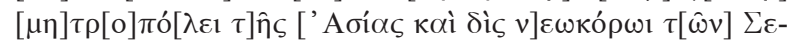

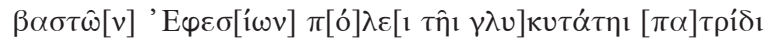

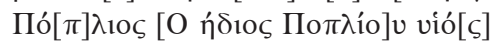

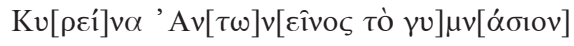

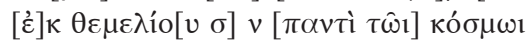

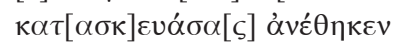

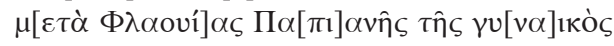

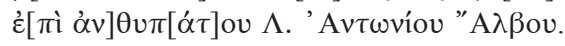

Es handelt sich dabei im wesentlichen um eine Wiederholung der nur sehr lückenhaft erhaltenen Fassung der Bauinschrift vom Epistyl der Hofhallen des Vediusgymnasiums (IvE 431).

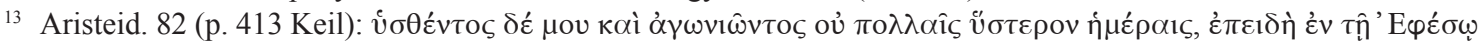

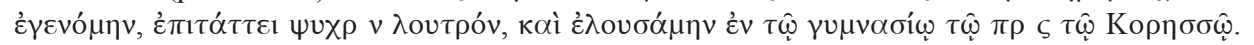

14 St. J. Friesen, Twice Neokoros. Ephesus, Asia and the Cult of the Flavian Imperial Family, EPRO 116 (1993) $134 \mathrm{ff}$. führt die Identifizierung der $\beta \alpha \lambda \alpha v \varepsilon \hat{\alpha} \alpha \tau \hat{\omega} v \sum \varepsilon \beta \alpha \sigma \tau \hat{\omega} v$ (IvE 1104. 1125) bzw. $\Sigma \varepsilon \beta \alpha \sigma \tau o \hat{v}$ (IvE 1155) mit dem Hafengymnasium überzeugend aus. P. Scherrer in: H. Thür (Hrsg.), »... und verschönerte die Stadt ...«. Ein ephesischer Priester des Kaiserkultes in seinem Umfeld, SoSchrÖAI 27 (1997) 112 Anm. 161 ergänzt gemäß IvE 621.

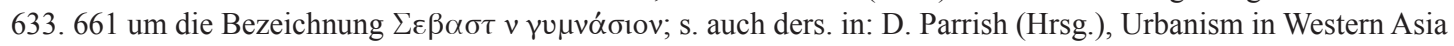
Minor. New Studies on Aphrodisias, Ephesos, Hierapolis, Pergamon, Perge and Xanthos, 45. Suppl. JRA (2001) 74; H. Halfmann, Städtebau und Bauherren im römischen Kleinasien. Ein Vergleich zwischen Pergamon und Ephesos, 43. Beih. IstMitt (2001) 42. - Für eine Umbauphase des Hafengymnasiums in der Mitte des 4. Jhs. n. Chr. ist darüber hinaus die spätantike Bezeichnung überliefert (IvE 1314. 1315): ... | atrio thermarum | Constantianarum $\mid$...

15 Allgemein zu den ephesischen Bad-Gymnasium-Komplexen: Maccanico (Anm. 11) 32-60; Fasolo (Anm. 11) 31-40; Farrington (Anm. 11) bes. 50 f.; Yegül (Anm. 2) 250 ff.; Nielsen (Anm. 2) 105 ff. - Zum Hafen-, Theaterund Ostgymnasium existieren darüber hinaus unpublizierte Manuskripte, die von F. Miltner in den 50er Jahren des 20. Jhs. verfaßt wurden und heute im Archiv des ÖAI in Wien verwahrt werden. 


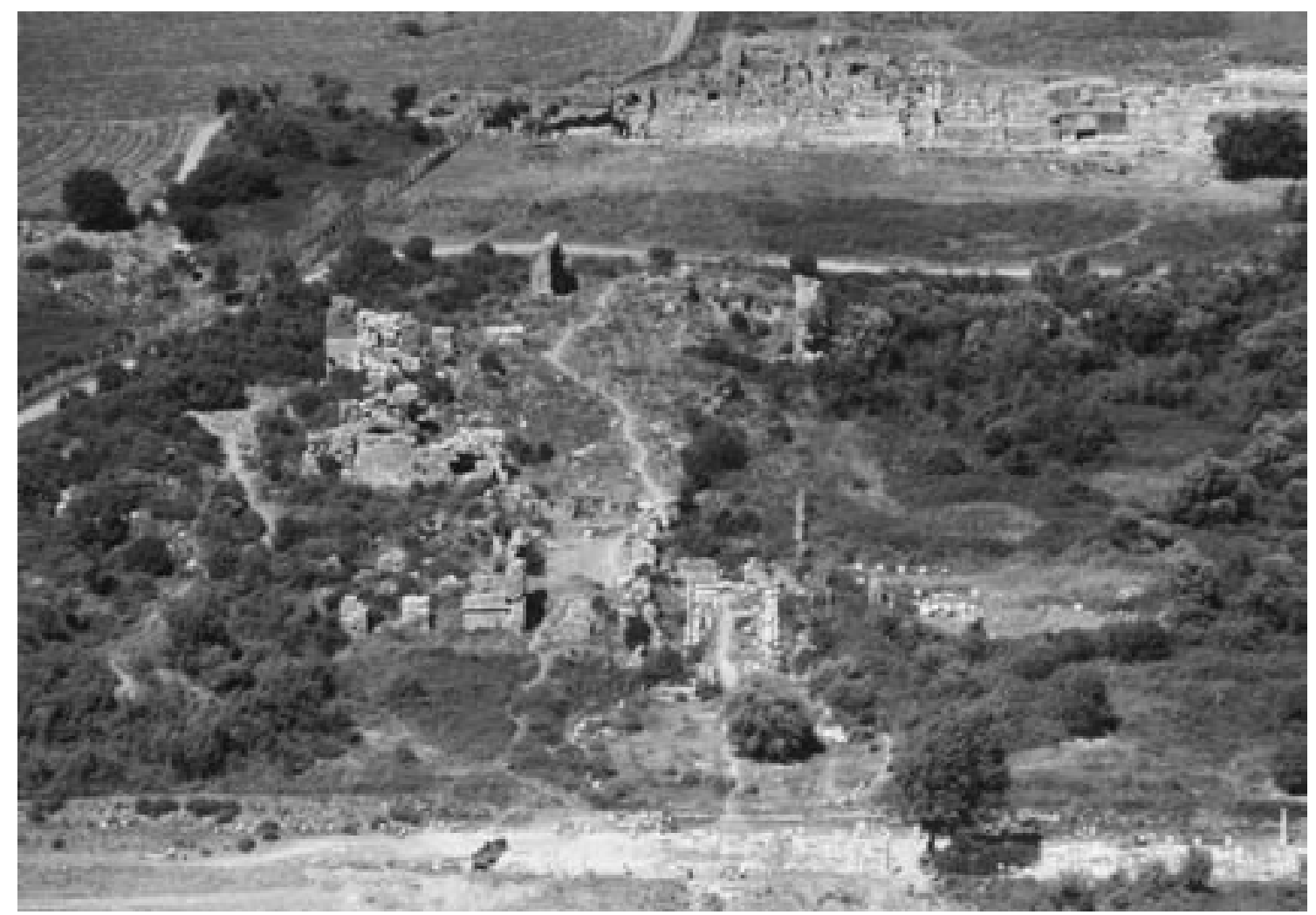

2 Ephesos. Hafengymnasium

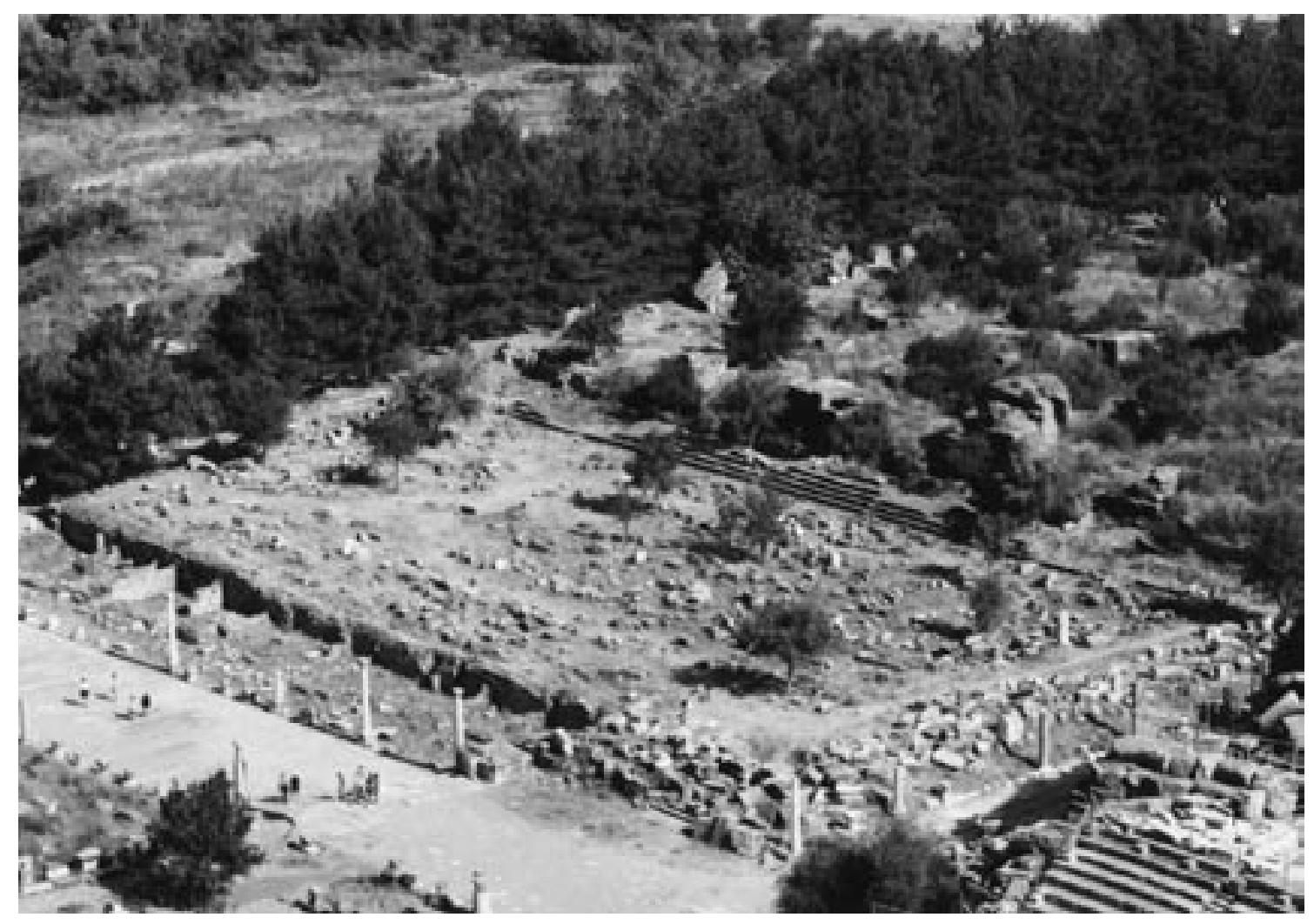

3 Ephesos. Theatergymnasium 
Den chronologisch ältesten Bad-Gymnasium-Komplex stellt das in domitianischer Zeit errichtete, nördlich der Arkadiane gelegene Hafengymnasium (Abb. 2) dar ${ }^{16}$. Der Bau ist in drei Teile gegliedert: die eigentlichen Thermen im Westen, die Palästra mit quadratischem Peristylhof $(90 \times$ $90 \mathrm{~m}$ ) in der Mitte und die sog. Verulanushallen $(200 \times$ $240 \mathrm{~m})$ im Osten. Bei der Bezeichnung 'Verulanushallen' handelt es sich um ei-

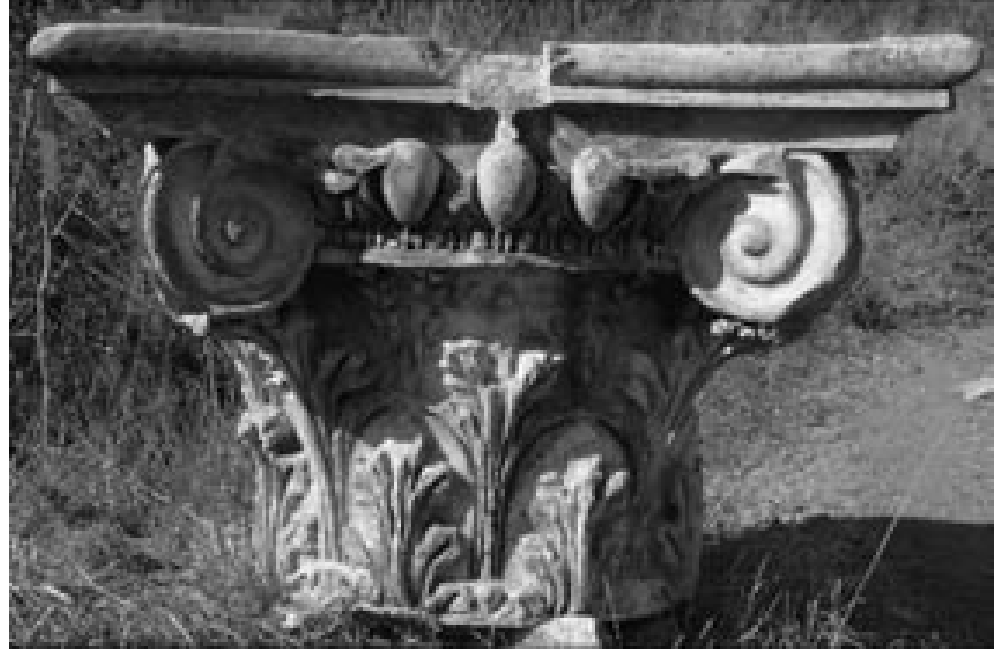

4 Kompositkapitell aus dem Hafengymnasium nen modernen Begriff, der auf die Stiftung marmorner Wandverkleidungsplatten in hadrianischer Zeit $(131 \mathrm{n}$. Chr.) durch den inschriftlich genannten Asiarchen Gaius Claudius Verulanus Marcellus (IvE 430) zurückzuführen ist. Die antike Bezeichnung dieser Hallen dürfte Xystos bzw. Xystoi gelautet haben ${ }^{17}$. Therme, Palästra und Xystos gehören einem einheitlichen, in kurzer Zeit realisierten Baukonzept $\mathrm{an}^{18}$. Die schon mehrmals geäußerte Vermutung, daß die Einrichtungen dieser Anlage noch vor der Ermordung des Domitian 96 n. Chr. der ephesischen Bevölkerung offenstanden, kann indes nicht eindeutig belegt werden. Die dafür ins Treffen geführte Stelle bei Philostrat über eine Vision des Apollonios von Tyana vom Tod des Domitian, die er im Xystos geäußert haben soll ${ }^{19}$, ist in diesem Zusammenhang nur von geringer Aussagekraft, da der kurz nach 217 n. Chr. verfaßten, romanhaften Auftragsbiographie, die vorwiegend auf lokalen mündlichen Überlieferungen basiert, nur bedingt Historizität beigemessen werden darf ${ }^{20}$. Als Stifter zumindest von Teilen der Anlage ist inschriftlich Tiberius Claudius Aristion bekannt (IvE 427), der den Marmor- bzw. Kaisersaal an der Südseite der Palästra errichten ließ.

Unmittelbar im Osten an die Verulanushallen anschließend wird in hadrianischer Zeit das sog. Theatergymnasium errichtet ${ }^{21}$ (Abb. 3). Aufgrund der Ähnlichkeit der Kapitelle dieser Anlage mit dem bereits im Hafengymnasium verwendeten Typus (Abb. 4) (2) $^{22}$ ann eine Datierung des nur sporadisch untersuchten Gebäudes kurz nach Fertigstellung des Hafengymnasiums postuliert werden. Grundrißtypologisch steht es mit seinen U-förmig umlaufenden Hallen im

16 Zum Hafengymnasium: J. Keil, ÖJh 28, 1933, Beibl. 14 ff.; O. Benndorf in: FiE I (1906) 181 ff.; ders., ÖJh 1, 1898, 62 ff.; R. Heberdey, ÖJh 7, 1904, Beibl. 43; Maccanico (Anm. 11) bes. 45 ff.; Friesen (Anm. 14) 121 ff.; Yegül (Anm. 2) 272 f.; Scherrer (Anm. 14:1997) 109 ff.

17 Die Anlage der dreischiffigen Säulenhalle mit parkähnlichem Hof entspricht den Vorgaben bei Vitr. 5, 11, 3 f. Zur antiken Bezeichnung: Vitr. 5, 11, 4: Haec autem porticus xystos apud Graecos vocitatur, ...

18 Vgl. RE Suppl. 12 (1970) 1610 f. s. v. Ephesos B (Alzinger). - Die Einwände gegen eine einheitliche Entstehung des Hafengymnasiums in domitianischer Zeit von Fasolo (Anm. 11) $34 \mathrm{ff}$. können nicht überzeugen.

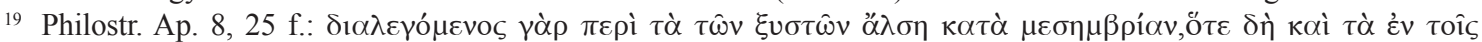

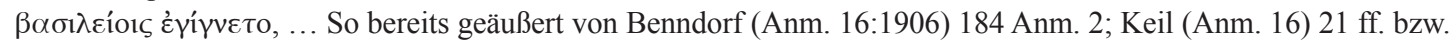
F. Miltner, Ephesos. Stadt der Artemis und des Johannes (1958) 48 f.; s. auch Scherrer (Anm. 14:1997) 112.

20 Deutlich hervorgehoben durch F. Grosso, Acme 7, 1954, 333 ff. - Zu Philostrat: G. Anderson, Philostratus. Biography and Belles Lettres in the Third Century A. D. (1986) bes. 227 ff.; RE XX 1 (1941) 136 ff. s. v. Philostratos (Solmsen).

${ }_{21}$ Zum Theatergymnasium: J. Keil, ÖJh 25, 1929, Beibl. 42 ff.; ders., ÖJh 26, 1930, Beibl. 18 ff.; ders., ÖJh 27, 1932, Beibl. 16 ff.; Maccanico (Anm. 11) bes. 42 f.; Yegül (Anm. 2) 279 ff.

22 Vgl. W.-D. Heilmeyer, Korinthische Normalkapitelle. Studien zur Geschichte der römischen Architekturdekoration, 16. Ergh. RM (1970) 94 Taf. 27, 3. Dazu auch G. A. Plattner, Ephesische Kapitelle des 1. und 2. Jhs. n. Chr. Form und Funktion kaiserzeitlicher Architekturdekoration in Kleinasien (ungedr. Diss. Wien 2003); ders., ÖJh 71, 2002, 237-249; V. M. Strocka, IstMitt 38, 1988, 302. 
Badblock der Therme des Ostgymnasiums sowie dem Bad-Gymnasium-Komplex in Alexandria Troas nahe. Letzterer wird als Stiftung des Herodes Atticus interpretiert und um $135 \mathrm{n}$. Chr. datiert $^{23}$. Die sekundäre Palästra mit ihrem $70 \times 30$ m großen, an drei Seiten von Säulenhallen umgebenen Hof ist überaus klein konzipiert und besitzt an ihrer Nordseite unmittelbar vor der Thermenfront eine tribünenförmige Konstruktion: Hinter vier Reihen von Sitzstufen befindet sich eine schräg ansteigende Ebene, die gemeinsam als Sitz- bzw. Stehplätze genützt werden konnte. Für die Palästra muß eine Umgestaltung in der zweiten Hälfte des 2. Jahrhunderts n. Chr. postuliert werden, die sich in der Bauornamentik deutlich ausdrückt.

Einen Sonderfall bildet das nördlich des Magnesischen Tores gelegene, ebenso nur teilweise untersuchte Ostgymnasium ${ }^{24}$ (Abb. 5). Palästra und Badblock gehören - wie im Theatergymnasium - unterschiedlichen Baukonzepten an. Während der Badblock mit seinen U-förmig umlaufenden Hallen zeitlich in der Mitte der ersten Hälfte des 2. Jahrhunderts n. Chr. anzusiedeln ist ${ }^{25}$, stellt die Palästra mit Kaisersaal und Auditorium an der West- bzw. Ostseite einen späteren Umbau dar. Dafür sprechen die bereits von F. Miltner in seinem unpublizierten Manuskript geäuBerten Argumente einer unterschiedlichen Mauertechnik ${ }^{26}$ sowie die überaus ungünstige Konstruktion der Nordfront der Palästra, die die für das entsprechende Raumklima essentiellen Fenster des Caldarium-Bereiches weitgehend verdeckte ${ }^{27}$. Neben grundrißtypologischen Erwägungen, wie etwa dem Verzicht auf absolute Symmetrie in der Palästra und dem auffällig klein konzipierten Peristylhof $(25 \times 30 \mathrm{~m})$, erfordert ein Vergleich der Bauornamentik, insbesondere der Kapitelle, zwingend eine Datierung des Palästrablocks in die zweite Hälfte des 2. Jahrhunderts n. Chr.: Die Verwendung des für den Neubau des Vediusgymnasiums in der Mitte des 2. Jahrhunderts hergestellten Typus von Kompositkapitellen mit Schnurstab (Abb. 6) belegt eine Datierung des Umbaus der Palästra in dieser Zeit ${ }^{28}$. Badblock und Palästra gehören somit nachweislich keinem einheitlichen Baukonzept an.

Über das ursprüngliche Aussehen der Palästra im Ostgymnasium kann ohne weitere feldarchäologische Untersuchungen nur noch gemutmaßt werden. J. Keil erwog - in Analogie zum Theatergymnasium - eine tribünenhafte Konstruktion an der Nordseite der Palästra, die insgesamt deutlich tiefer (um etwa $2 \mathrm{~m}$ ) als das Niveau der jüngeren Palästra gelegen wäre ${ }^{29}$. Auf diese Weise wäre auch das Caldarium mit genügend Licht versorgt gewesen, da die Bogenfenster der Südfront des Bades so nicht mehr durch das davorliegende Peristyl verdeckt worden wären.

$\mathrm{Da} ß$ es sich bei der Finanzierung des Umbaus der Palästra im Ostgymnasium um eine Stiftung des Sophisten T. Flavius Damianus ${ }^{30}$ und seiner Gattin Vedia Phaedrina ${ }^{31}$ handelte, ist

23 Zum Bad-Gymnasium-Komplex in Alexandria Troas: R. Koldewey, AM 9, 1884, 36-48; A. C. G. Smith, AnatSt 29, 1979, 23-50; Krencker (Anm. 2) 285 ff.; Yegül (Anm. 2) 282.

${ }^{24}$ Zum Ostgymnasium: Keil (Anm. 21:1932) 25 ff.; ders. (Anm. 16) 6 ff.; Maccanico (Anm. 11) bes. 43 ff.; Yegül (Anm. 2) 279 ff.

25 Vgl. die typologisch verwandten Konstruktionen im Theatergymnasium bzw. im Bad-Gymnasium-Komplex von Alexandria Troas.

26 Besonders deutlich beim Vergleich des Mauerwerks der Nordmauer der umlaufenden Hallen mit den Mauern des Kaisersaals. Darüber hinaus kann die Verwendung von Spolien, wie beispielsweise am Auditorium der Palästra, im gesamten Badblock nicht beobachtet werden.

27 s. dazu auch H. Manderscheid, Die Skulpturenausstattung der kaiserzeitlichen Thermenanlagen, MAR 15 (1981) $14 \mathrm{f}$.

28 Vgl. Plattner (Anm. 22:2003); ders. (Anm. 22:2002) 237-249. - Zur Gleichsetzung der Bauhütten s. bereits P. Scherrer in: H. Koester (Hrsg.), Ephesos. Metropolis of Asia. An Interdisciplinary Approach to its Archaeology, Religion, and Culture, Harvard Theological Studies 41 (1995) 14.

29 Keil (Anm. 21:1932) 32 ff. und F. Miltner (unpubl.).

30 Zur Person u. a.: PIR² F 253; D. Knibbe in: ders. - G. Langmann, Via Sacra Ephesiaca I, BerMatÖAI 3 (1993) 56 f. mit zahlreichen Literaturangaben; H. Halfmann, Die Senatoren aus dem östlichen Teil des Imperium Romanum bis zum Ende des 2. Jahrhunderts n. Chr., Hypomnemata 58 (1979) 40. 137. 168. 192; C. Schulte, Die Grammateis von Ephesos. Schreiberamt und Sozialstruktur in einer Provinzhauptstadt des römischen Kaiserreiches, Heidelberger althistorische Beiträge und epigraphische Studien 15 (1994) 184 ff. Nr. 116; E. Groag, ÖJh 10, 1907, 295 ff.

31 Zur Person u. a.: RE VIII A 1 (1955) 567 f. Nr. 5 s. v. Vedius (Keil); M.-T. Raepsaet-Charlier, Revue international des droits de l'antiquité 30, 1983, 185-192. 


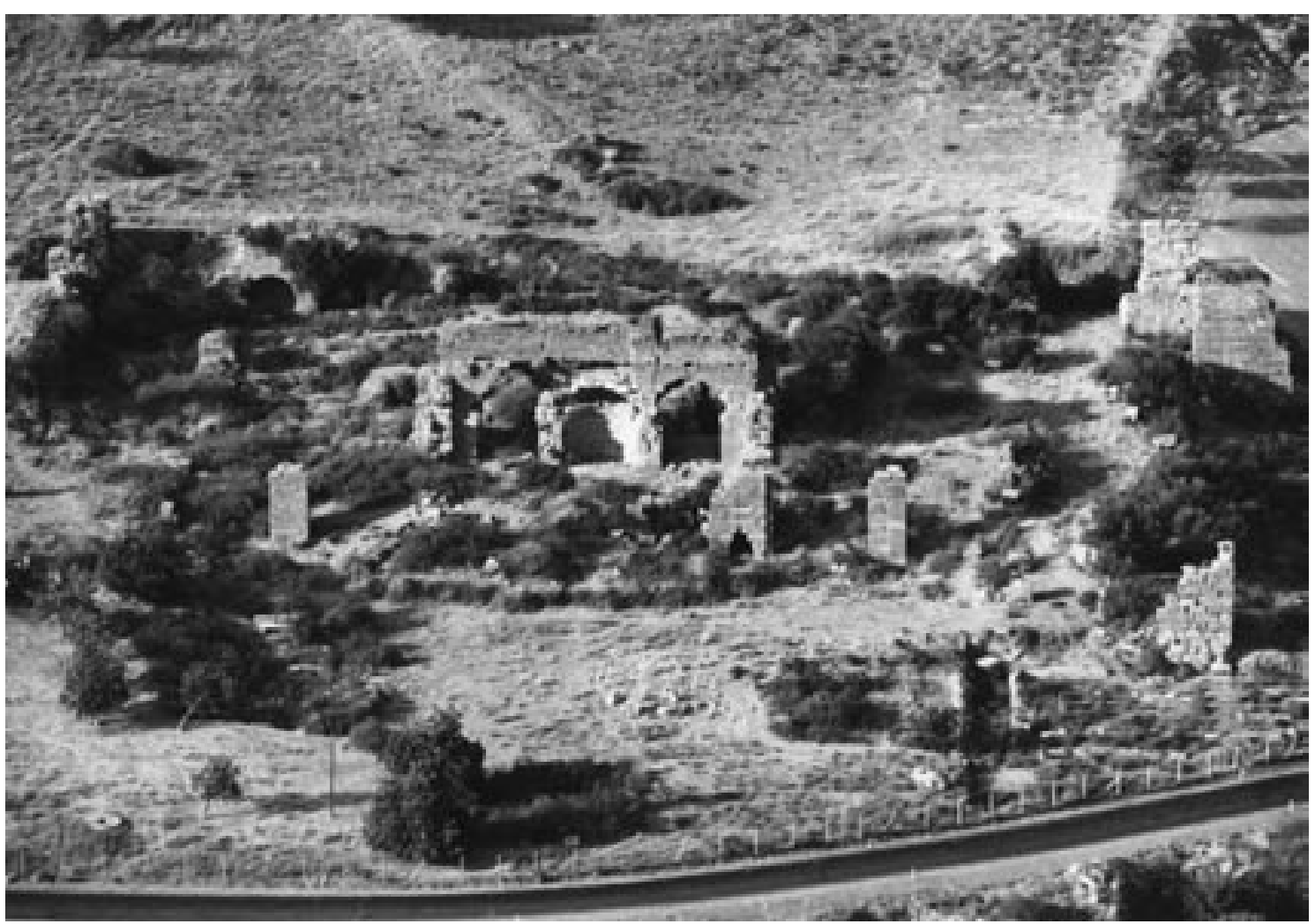

5 Ephesos. Ostgymnasium

nicht belegt, zumal die Identifizierung der in der Stifterinschrift genannten Person ] $v \eta \varsigma^{~ ' A} \nu \tau \omega[$

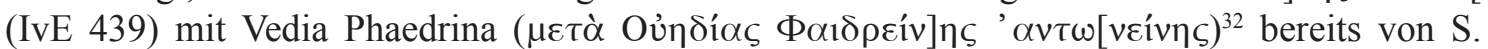
Dillon zu Recht falsifiziert wurde ${ }^{33}$. In keiner der ephesischen Inschriften, in denen Vedia Phaedrina genannt wird, führt sie nämlich das Cognomen 'Antonina' oder 'Antoniniana'34.

Das laut seiner Bauinschrift (IvE 431. 438) ${ }^{35}$ zwischen 147 und 149 n. Chr. eingeweihte, von M. Claudius P. Vedius Antoninus Phaedrus Sabinianus ${ }^{36}$ gestiftete Vediusgymnasium (Abb. 7) besitzt östlich an den Badblock anschließend eine Palästra mit einem etwa $40 \times 50 \mathrm{~m}$ großen, an allen vier Seiten von Säulenhallen umgebenen $\operatorname{Hof}^{37}$. Über die Südseite des Peristyls konnte das Gebäude durch ein dreiachsiges Propylon betreten bzw. verlassen werden. Der Hof selbst konnte vom Niveau der Hallen, die wie die dahinterliegenden Räume schon zur Bauzeit mit Mosaiken ausgestattet waren, über zwei Stufen erschlossen werden. In den Badblock integriert,

32 Gemäß Keil (Anm. 21:1932) 31 f. Anm. 3. Übernommen von Alzinger (Anm. 18) 1614 und F. Miltner (unpubl.), dann weitgehend in der modernen Literatur rezipiert; so etwa von Manderscheid (Anm. 27) 14 f.; Yegül (Anm. 2) 423; ders. (Anm. 11:1982) 11.

33 S. Dillon, JRA 9, 1996, 272. So auch Halfmann (Anm. 14) 79 Anm. 262 und J. Rumscheid, Kranz und Krone. Zu Insignien, Siegespreisen und Ehrenzeichen der römischen Kaiserzeit, IstForsch 43 (2000) $34 \mathrm{ff}$.

34 Vgl. IvE 47. 678. 733. 2100. 3081.

35 Vgl. Anm. 12.

36 Zur Person u. a.: M. Steskal, Tyche 16, 2001, 177-188; Halfmann (Anm. 30) 169 f. Nr. 84b; Schulte (Anm. 30) 170 ff. Nr. 92; RE VIII A 1 (1955) 566 s. v. Vedius (Keil); A. Kalinowski, Phoenix 56, 2002, 109 ff.; M. D. Campanile, I sacerdoti del Koinon d'Asia (I sec. a.C. - III sec. d.C.). Contributo allo studio della romanizzazione delle élites provinciali nell'Oriente greco, Studi ellenistici 7 (1994) 113 ff.

37 Zum Vediusgymnasium: M. Steskal - M. La Torre, ÖJh 70, 2001, 223-246; J. Keil, ÖJh 24, 1929, Beibl. 20 ff.; ders. (Anm. 21:1929) 21 ff.; ders. (Anm. 21:1930) 17 ff.; F. Miltner, ÖJh 42, 1955, Beibl. 23 ff.; Krencker (Anm. 2) 287 f.; Maccanico (Anm. 11) bes. 38 ff.; Yegül (Anm. 2) 282 ff. 


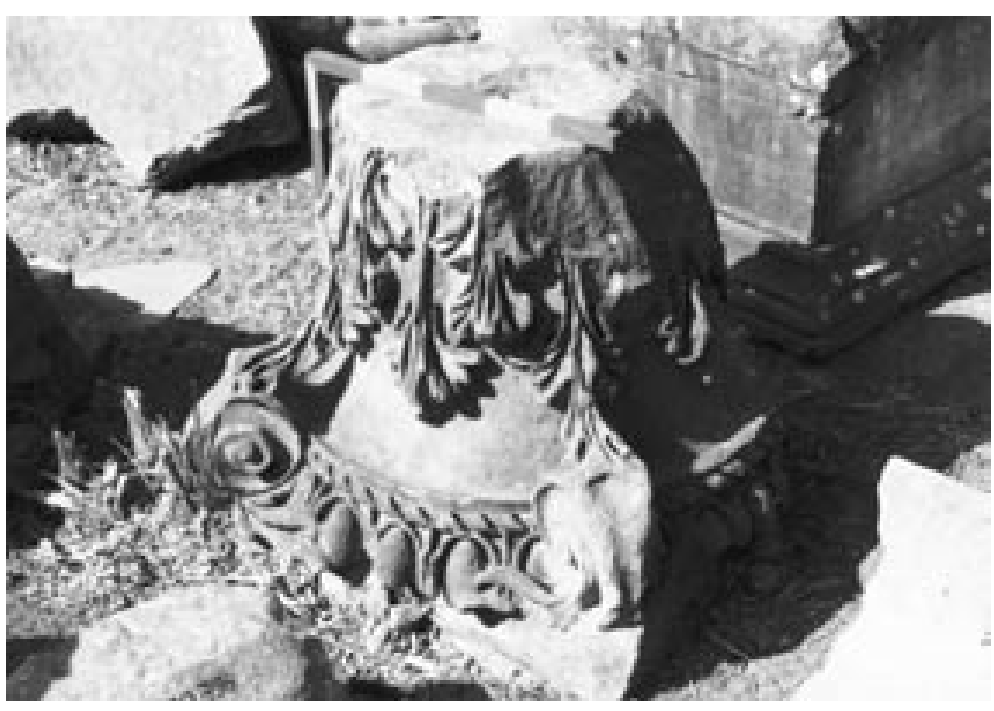

6 Kompositkapitell mit Schnurstab aus dem Vediusgymnasium

jedoch funktional zur Palästra gehörig, öffnet sich an der Ostseite der Therme eine u. a. als Kaisersaal zu interpretierende Exedra. $\mathrm{Ob}$ die archäologisch unerforschte Fläche östlich des Vediusgymnasiums einen Xystos - formal ähnlich den Verulanushallen - besa $\beta$, kann bis dato nicht bestätigt werden. Die frappierende Ähnlichkeit mit dem jüngeren Bad-Gymnasium-Komplex in Sardes, in dem der Grundriß des Vediusgymnasiums in monumentalisierter und noch ausgefeilterer

Form wiedergegeben wird $^{38}$, läßt vermuten, daß derselbe Architekt für seine Errichtung verantwortlich zeichnet oder zumindest die Baupläne rezipiert wurden.

Neben dem Vediusgymnasium besitzen auch das Hafen- und Ostgymnasium sog. Kaisersäle, die einen integrativen Bestandteil der Palästren darstellen ${ }^{39}$. Die zuletzt von J. Rumscheid geäußerte Kritik an der Zuweisung der Kaisersäle zum Kaiserkult kann in diesem Kontext nicht bestehen ${ }^{40}$. Die Freilegung des noch in situ angetroffenen, marmornen Altares vor der Mittelnische des Kaisersaales im Vediusgymnasium, das in der zweifach erhaltenen Bauinschrift (IvE 431. 438) explizit als Weihung an Antoninus Pius bezeichnet wird, gilt in diesem Zusammenhang als eindeutiges Indiz ${ }^{41}$. Die zusätzliche Nennung der Göttin Artemis und der Stadt Ephesos muß vor diesem Hintergrund als formelhafte Wendung, aber auch als Betonung des Stiftergedankens betrachtet werden. Die ausdrückliche Nennung der Weihung an die Stadt diente der Hervorhebung der Munifizenz des Vediers.

Die Kaisersäle in den ephesischen Bad-Gymnasium-Komplexen müssen - zumindest zum Zeitpunkt ihrer Errichtung - als Orte des Kaiserkultes und der Selbstdarstellung der Stifter verstanden werden. Eine über diese Nutzung hinausgehende Multifunktionalität dieser Säle, etwa als Orte der Repräsentation, Versammlungs- oder Clubsäle etc., soll in diesem Zusammenhang nicht ausgeschlossen werden ${ }^{42}$.

Der Umbau der Palästra im Ostgymnasium in der zweiten Hälfte des 2. Jahrhunderts n. Chr., ihre Verkleinerung, die Errichtung eines sog. Auditoriums an ihrer Ostseite und die Pflasterung

38 Vgl. Yegül (Anm. 11:1986) Abb. 378. 379.

39 Im Theatergymnasium ist der Kaisersaal in den Badblock eingebunden. - Zur Zuweisung der Kaisersäle zum Kaiserkult s. vor allem Yegül (Anm. 11:1982) 7-31; Manderscheid (Anm. 27) $36 \mathrm{f}$.

40 Rumscheid (Anm. 33) 44 f. Bezugnehmend auf S. R. F. Price, Rituals and Power. The Roman imperial cult in Asia Minor (1984) 144 Anm. 34. - Zum Kaiserkult allgemein s. außerdem Friesen (Anm. 14) 142 ff.; M. Wörrle, Stadt und Fest im kaiserzeitlichen Kleinasien. Studien zu einer agonistischen Stiftung aus Oinoanda, Vestigia 39 (1988) 101 ff. 216 ff. 248 ff. Zu den mannigfaltigen Formen des Kaiserkultes s. I. Gradel, Emperor Worship and Roman Religion (2002).

${ }^{41} \mathrm{Zu}$ den Fundumständen s. Keil (Anm. 37:1929) 35 f.

42 Monokausale Erklärungsmodelle können in diesem Kontext nicht bestehen. Jede diesbezügliche Interpretation bildet nur eine Momentaufnahme. Im Laufe der Jahrhunderte unterliegen die Bedeutung und Funktion der Kaisersäle einem kontinuierlichen Wandel. So kann etwa für das Vediusgymnasium nach seiner Umgestaltung im 1. Viertel des 5. Jhs. n. Chr. (vgl. Anm. 43) eine Interpretation seines Kaisersaals als Ort des Kaiserkultes naturgemäß ausgeschlossen werden. - C. Schneider, Die Musengruppe von Milet, Milesische Forschungen 1 (1999) 66 ff. interpretiert die Kaisersäle neuerdings als Empfangsräume der Personen von Rang und Vermögen, vergleichbar mit einer 'V.I.P.-Lounge' heutiger Zeit. 


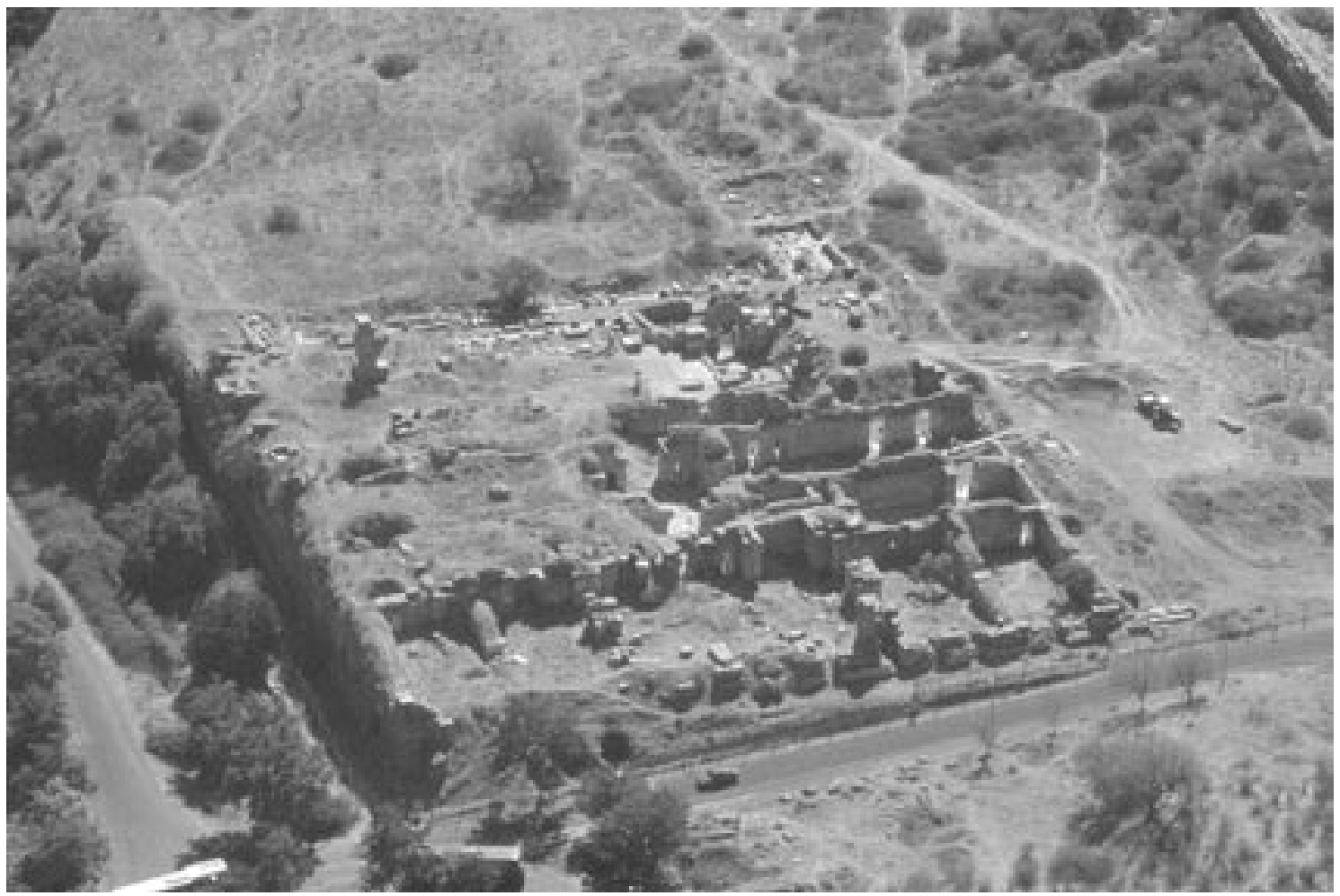

7 Ephesos. Vediusgymnasium

des Peristylhofes mit Marmor verdeutlichen einen allgemeinen Funktionswandel. Die Palästra scheint mit diesem veränderten architektonischen Konzept ihren gymnischen Charakter völlig verloren zu haben. Sportliche Betätigung in größerem Rahmen kann im Bereich der mit Marmor gepflasterten Palästra ab diesem Zeitpunkt ausgeschlossen werden. Hauptaugenmerk wird vielmehr auf intellektuelle Interaktion sowie musisch-philosophische Ausbildung gelegt, wie durch die Einrichtung des Vortragssaales eindrucksvoll dokumentiert wird.

Ein ähnlicher Funktionswandel kann in der basilica thermarum (Raum III) im Vediusgymnasium baulich nachgewiesen werden. Wie bei Ausgrabungen im Sommer 2001 in Raum IIIb festgestellt werden konnte, wurden in der zweiten Ausstattungsphase die Wasserbecken in den Exedren durch Sitzbänke ersetzt ${ }^{43}$ (Abb. 8). Auf eine Nutzung dieser ursprünglich vielleicht auch für sportliche Aktivitäten zur Verfügung stehenden basilica thermarum als Vortrags- oder Lesesaal bzw. als Ort der Diskussion kann somit geschlossen werden ${ }^{44}$. Daß es sich bei dem zwischen 147 und $149 \mathrm{n}$. Chr. eingeweihten Vediusgymnasium zunächst um eine multifunktionale Anlage mit sportlichen und intellektuellen Aspekten gehandelt haben wird, muß zwar in Betracht gezogen werden, von einem von Anfang an geplanten Schwerpunkt als Bildungs- und Ausbildungszentrum oder als Ort des philosophischen Diskurses kann aber ausgegangen werden. Dies verdeutlicht in besonderem Maße die programmatische Ausstattung der Anlage mit Her-

${ }^{43}$ Nach Analyse des Fundmaterials, für die Frau S. Ladstätter gedankt sei, kann diese Umbauphase, in der der Raum auch mit einem Marmorpflaster, die Hallen des Peristyls der Palästra mit neuen polychromen Mosaikböden und der Kaisersaal mit einem neuen opus sectile-Paviment ausgestattet wurden, in das 1. Viertel des 5. Jhs. n. Chr. datiert werden.

44 Zur Funktion der basilicae thermarum s. Nielsen (Anm. 2) 144 ff. 162. - Der von Yegül (Anm. 11:1986) 149 Anm. 20 geäußerten Vermutung, daß Raum III des Vediusgymnasiums ursprünglich als Apodyterium genutzt wurde, kann aufgrund der Grabungsergebnisse des Jahres 2001 widersprochen werden. Die zur Primärausstattung gehörigen Wasserbecken in den Exedren von Raum IIIb und das Fehlen von Sitzbänken in dieser Phase sprechen deutlich gegen eine Identifizierung als Apodyterium. 


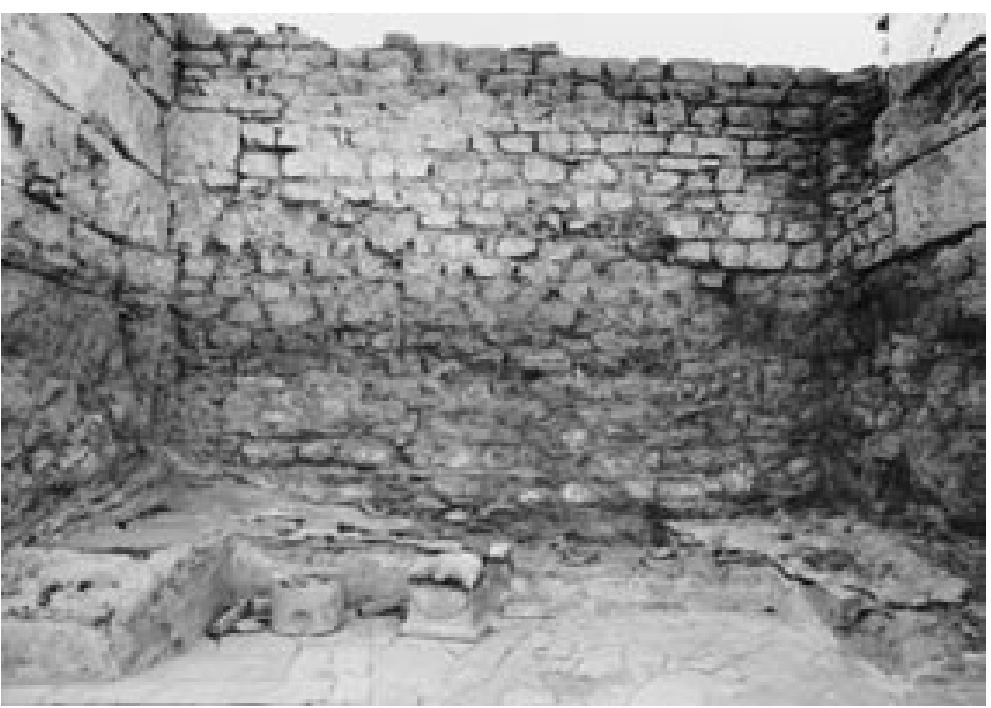

8 Vediusgymnasium. Südwestliche Exedra in Raum IIIb $\mathrm{men}^{45}$. Die beiden im Propylon zur Palästra aufgestellten Hermen des Herakles bzw. Hermes ${ }^{46}$, die als Schutzgötter der Gymnasien traditionell 'Aufgabenfelder' im athletisch-sportlichen (Herakles) und musisch-philosophischen Bereich (Hermes) wahrnahmen $^{47}$, müssen im Fall des Vediusgymnasiums anders interpretiert werden. Herakles ist im Propylon nämlich nicht als athletischer, kräftig gebauter Held, sondern in fortgeschrittenem Alter im 'Philosophentypus' dargestell $t^{48}$. Daß er sich auf diese Art primär als Schutzgott der Athleten präsentierte, kann ausgeschlossen werden. Interessant ist in diesem Zusammenhang, daß eine weitere im Apodyterium (Raum VI), neben der Tür zu Raum VIII aufgestellte kolossale Herme nicht etwa, wie bisher vermutet, Herakles verkörperte $^{49}$, sondern als Apollo-Ariadne-Typus oder - neben der auf der anderen Seite der Tür positionierten Herme des Hermes Propylaios ${ }^{50}$ - als nochmalige Hermes-Darstellung interpretiert werden $m u \beta^{51}$. Das Hermen-Programm impliziert somit die vorrangige Art der Nutzung der Palästra: Sie diente in erster Linie als Ort der Bildung und intellektuellen Interaktion. Die unmittelbare Nähe zum südlich gelegenen Stadion ermöglichte zudem eine Auslagerung der sportlichen Aktivitäten. Eine ähnliche Situation läßt sich in Laodikeia ${ }^{52}$, Nysa (Ostgymnasium) $)^{53}$, Aizanoi $^{54}$ und Milet (Faustinathermen) ${ }^{55}$ nachweisen, wo wie in Ephesos ein Bad-GymnasiumKomplex oder eine Therme neben dem benachbarten Stadion errichtet wurde ${ }^{56}$.

Eine vergleichbare Konstellation kann für das Hafen- und Theatergymnasium in Ephesos konstatiert werden: Mit den Verulanushallen stand ein multifunktional nutzbares Areal von nicht unbeträchtlichen Ausmaßen zur Verfügung, das zur körperlichen und geistigen Ertüchtigung

45 Für den freundlichen Hinweis danke ich Frau R. Hanslmayr, die mit der Bearbeitung der Hermen aus dem Vediusgymnasium betraut ist und mir Einsicht in das noch unpublizierte Manuskript gewährte. Bei den vier vorgestellten Hermen handelt es sich jeweils um Objekte der Primärausstattung; s. R. Hanslmayr, Hermen aus Ephesos, FiE X 1, 1 (in Vorbereitung). - Zur Aufstellung von Hermen in Gymnasien und Palästren allgemein: B. Rückert, Die Herme im öffentlichen Leben der Griechen (1998) $126 \mathrm{ff}$.

46 s. Manderscheid (Anm. 27) 89 Nr. 179 und 188. Die bei ihm mit dem Vermerk »verschollen? « angeführten Hermen aus dem Vediusgymnasium konnten durch R. Hanslmayr wieder identifiziert werden. Beide befinden sich heute im Efes Müzesi Selçuk (Mantelherme des Hermes: Inv. 408; fellbekleidete Herme des Herakles: ohne Inv.).

47 Besonders deutlich ausgeführt bei Delorme (Anm. 2) 339 ff.; s. auch Ch. Vorster, KölnJb 21, 1988,10 ff.; Wacker (Anm. 3) $114 \mathrm{f}$.

48 Vgl. Hanslmayr (Anm. 45).

49 So u. a. vermutet von Manderscheid (Anm. 27) 89 Nr. 178.

50 Manderscheid (Anm. 27) 89 Nr. 180. Die Herme befindet sich heute in Izmir, Arkeoloji Müzesi Inv. 675.

51 Zur Identifizierung als Apollo s. D. Willers, JdI 82, 1967, 37 ff.; heute im Efes Müzesi Selçuk, ohne Inv.

52 Yegül (Anm. 2) 258. 278. 416 ff.; G. E. Bean, Turkey Beyond the Maeander (1971) 253.

53 W. von Diest, Nysa ad Maeandrum, 10. Ergh. JdI (1913) $44 \mathrm{ff}$.

${ }^{54}$ R. Naumann, AA 1980, 123-136; ders., AA 1982, 345-356; R. Naumann - F. Naumann, AA 1984, 453-456.

55 F. Krischen in: A. von Gerkan - F. Krischen, Thermen und Palaestren, Milet I 9 (1928) 50 ff.; Krencker (Anm. 2) 284 f.; G. Kleiner, Die Ruinen von Milet (1968) 101-109.

56 Vgl. Yegül (Anm. 2) $308 \mathrm{f}$. 
frequentiert werden konnte ${ }^{57}$. Dies scheint besonders in Anbetracht der überaus klein konzipierten Palästra des Theatergymnasiums, die für groß angelegte sportliche Aktivitäten ungeeignet gewesen ist, von Bedeutung. Die grundsätzliche Verlagerung des Interesses von sportlicher zu geistiger Ausbildung und Betätigung spiegelt die Tatsache wider, daß der im dritten Viertel des 3. Jahrhunderts n. Chr. zerstörte Xystos mit seinen überdachten Laufbahnen, größter Sportplatz der Stadt und wahrscheinlicher Austragungsort der ephesischen Olympien und anderer Agone ${ }^{58}$, nicht mehr aufgebaut ${ }^{59}$, sondern von einfachen Wohnquartieren überbaut wird, während die Thermen in der Mitte des 4. Jahrhunderts als thermae Constantianarum (IvE 1314. 1315) wiedererrichtet werden. Dieser Umstand korreliert mit einem generellen Rückgang an abgehaltenen Agonen ab der zweiten Hälfte des 3. Jahrhunderts n. Chr., der nicht zuletzt mit einem signifikanten wirtschaftlichen Niedergang einhergeht ${ }^{60}$.

Die geringe Größe der Palästren in den ephesischen Bad-Gymnasium-Komplexen bedingte offenbar von jeher eine Auslagerung groß angelegter sportlicher Aktivitäten. Lediglich für das Ostgymnasium kann ein entsprechender Sportplatz bis dato nicht nachgewiesen werden. Der Funktionswandel zur vorrangig intellektuellen Bildungs- und Ausbildungsstätte kann am Umbau der Palästra im Ostgymnasium, an den baulichen Veränderungen im Vediusgymnasium und am Verzicht auf einen Wiederaufbau der Verulanushallen deutlich abgelesen werden.

Ein in der römischen Kaiserzeit allgemein feststellbares und wachsendes Desinteresse an sportlicher Betätigung nur um der ganz in griechischer Tradition stehenden körperlichen Ertüchtigung willen wurde schon mehrfach betont ${ }^{61}$. Das diesbezügliche Urteil römischer Autoren ist - bereits seit den Zeiten der Republik - durchwegs eindeutig ${ }^{62}$, so schreibt etwa Cicero ${ }^{63}$ : $» W i e$ unsinnig sind doch die Übungen der Jugend in den Gymnasien!« Ihm folgt Seneca ${ }^{64}$ : »Es ist töricht, mein Lucilius, und für einen Menschen mit geistigen Interessen überaus unpassend, den Körper durch athletische Übungen zu kräftigen. « Plutarch berichtet im 1. Jahrhundert n. Chr. ${ }^{65}$ :

57 Yegül (Anm. 2) 308: »..., the vast triple-colonnaded enclosure $(200 \times 240 \mathrm{~m})$ might have been the most impressive and commodious sports arena of the Classical world.«

58 s. Friesen (Anm. 14) 128 ff. und Scherrer (Anm. 14:1997) 112. - Die Veranstaltung von Agonen, die einem breiten Publikum dargeboten werden sollten, in den Palästren der ephesischen Bad-Gymnasium-Komplexe scheint nicht zuletzt aufgrund der eindeutigen Quellenlage und der räumlichen Gegebenheiten wenig überzeugend; s. etwa J. Auinger in: B. Asamer - W. Wohlmayer (Hrsg.), Akten des 9. Österreichischen Archäologentages, Salzburg 2001 (2003) 15-19, der in der übrigen Argumentation aber gefolgt werden kann.

59 s. Benndorf (Anm. 16:1906) 184; C. Foss, Ephesus after Antiquity: A late Antique, Byzantine and Turkish City (1979) 60 und Yegül (Anm. 2) 313, die die Zerstörung mit dem Goteneinfall in den 60er Jahren des 3. Jhs. n. Chr. in Verbindung bringen. Tatsächlich wird für die massiven Zerstörungen auch eine Naturkatastrophe wie etwa ein Erdbeben in Betracht zu ziehen sein. Vgl. in diesem Zusammenhang St. Karwiese in: Lebendige Altertumswissenschaft. Festschrift H. Vetters (1985) 126-131, der ein schweres Erdbeben in dieser Region ins Jahr 262 n. Chr. datiert. Kritisch dazu: S. Ladstätter in: F. Krinzinger (Hrsg.), Das Hanghaus 2 von Ephesos. Studien zu Baugeschichte und Chronologie, AForsch 7, DenkschrWien 302 (2002) 26 ff. - Gegen eine verbindliche Datierung der Zerstörung des Hafengymnasiums spricht sich Alzinger (Anm. 18) 1611 aus.

60 Ausführlich begründet anhand des epigraphischen Materials von Aphrodisias bei Roueché (Anm. 9) 2 ff. 137. Die Autorin weist aber trotz dieses Rückgangs, den sie auf eine fortschreitende Geldentwertung in dieser Zeit zurückführt, auf die Kontinuität der Agone bis in die Spätantike hin; vgl. in diesem Zusammenhang die kritische Studie von I. Weiler, Grazer Beiträge 12/13, 1985/1986, 235-263 zum Ende der antiken Olympischen Spiele mit zahlreichen Literaturangaben. s. auch H. V. Herrmann, Gymnasium 80, 1973, bes. 196 ff.

${ }^{61}$ So z. B. von Glass (Anm. 2:1988) 157; Maccanico (Anm. 11) 55; M. Luni, QuadALibya 8, 1976, 230; A. Hönle, AW 14, 1983, 56; G. Lukas, Die Körperkultur in frühen Epochen der Menschheitsentwicklung (1969) 145 ff.; K. Palaeologos in: The International Olympic Academy. Eleventh Session. Athen (1971) 54-70.

62 Vgl. in diesem Zusammenhang St. Müller, Das Volk der Athleten. Untersuchungen zur Ideologie und Kritik des Sports in der griechisch-römischen Antike, Bochumer altertumswissenschaftliches Colloquium 21 (1995) bes. $212 \mathrm{ff}$.

${ }_{63}$ Cic. rep. 4, 4: Iuventutis vero exercitatio quam absurda in gymnasiis!

${ }^{64}$ Sen. epist. 15: Stulta est enim, mi Lucili, et minime conveniens litterato viro occupatio exercendi lacertos et dilatandi cervicem ac latera firmandi; ...

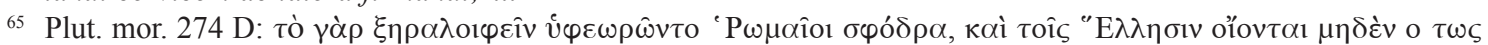

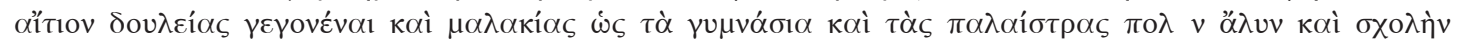

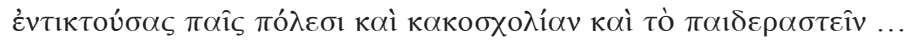


»Das >Trockensalben` war den Römern immer schon sehr suspekt, und auch heute noch glauben sie, daß bei den Griechen nichts so sehr verantwortlich sei für Knechtschaft und Verweichlichung wie die Gymnasien und Palästren, die den Städten viel Untätigkeit und Freizeit gebracht hätten, Vergeudung von Zeit und die Päderastie ...« Aber auch Tacitus macht kein Hehl aus seiner Abneigung gegenüber sportlicher Aktivität ${ }^{66}: »$ Die Sitten der Väter würden immer mehr untergraben durch fremdartige Ausgelassenheit, damit man in Rom ja alles, was verdorben und verderbenbringend sei, sehen könne, und damit die Jugend durch den Besuch der Sportplätze, durch Müßiggang und schändliche Liebesabenteuer zugrundegerichtet werde.« Vitruv wiederum zeigt sich über die seiner Meinung nach übertriebenen Ehrungen erfolgreicher griechischer Athleten durch frühere Generationen befremdet ${ }^{67}: »$ Wenn ich das also betrachte, muß ich mich wundern, warum die gleichen ehrenvollen Auszeichnungen und sogar noch größere nicht auch den Schriftstellern zuteil geworden sind, die der ganzen Welt für alle Zeiten unendlich gute Dienste leisten. Es wäre nämlich würdiger gewesen, diese Schriftsteller zu ehren, weil die Sportler durch Training zwar ihre eigenen Körper stählen, die Schriftsteller aber nicht nur ihren eigenen Geist, sondern das allgemeine Geistesleben bereichern, da sie durch ihre Bücher Wissen bereitstellen, damit man durch sie Kenntnisse erwirbt und den Geist schärft.«

Die Ressentiments der intellektuellen Oberschicht gegenüber der besonders von der griechischen Bevölkerung getragenen sportlichen Aktivität werden auch in der einfachen Bevölkerung Widerhall gefunden haben ${ }^{68}$.

Wenn die angeführten Stellen auch auf Rom Bezug nehmen, so muß das Desinteresse an körperlicher Betätigung als Selbstzweck - einer zutiefst griechischen Tradition - in den östlichen Provinzen, die sich anhand architektonischer bzw. konzeptueller Veränderungen an den ephesischen Bad-Gymnasium-Komplexen seit der zweiten Hälfte des 2. Jahrhunderts n. Chr. dokumentieren lassen, als Teilaspekt einer fortgeschrittenen Akkulturation einer nach mehr als 300 Jahren römischer Herrschaft weitgehend romanisierten Gesellschaft verstanden werden ${ }^{69}$. Daß die Kritik an sportlicher Aktivität auch in den östlichen Provinzen durchaus lebhaft war, spiegelt sich vor allem im Protreptikos des Galen, verfaßt in der zweiten Hälfte des 2. Jahrhunderts n. Chr., wider ${ }^{70}$.

Nicht zuletzt sei in diesem Zusammenhang auf die ausgeprägte Aburteilung des Sports durch die Kirchenschriftsteller der späteren Kaiserzeit verwiesen ${ }^{71}$, vor allem vertreten durch Tertullian am Beginn des 3. Jahrhunderts ${ }^{72}$ sowie Novatian in der Mitte des 3. Jahrhunderts n. Chr. ${ }^{73}$. In einer Zeit, in der das Interesse an den Disziplinen des klassischen Pentathlon schon weitgehend verloren war, galt die schärfste Kritik dem Faustkampf und dem Pankration, die in ständiger

66 Tac. ann. 14, 20: Ceterum abolitos paulatim patrios mores funditus everti per accitam lasciviam, ut, quod usquam corrumpi et corrumpere queat, in urbe visatur degeneretque studiis externis iuventus, gymnasia et otia et turpes amores exercendo, ...

${ }^{67}$ Vitr. 9, praef. 1: Cum ergo id animadvertam, admiror, quid ita non scriptoribus eidem honores etiamque maiores sint tributi, qui infinitas utilitates aevo perpetuo omnibus gentibus praestant. Id enim magis erat institui dignum, quod athletae sua corpora exercitationibus efficiunt fortiora, scriptores non solum suos sensus, sed etiam omnium, cum libris ad discendum et animos exacuendos praeparant praecepta.

68 Weitere Belegstellen bei Müller (Anm. 62) 220 Anm. 45. Daß es sich bei diesen Stellen partiell um Rückgriffe auf ältere Autoren wie etwa Xenophanes von Kolophon (Xenophan. Fr. 2 DK, 11 f.) handelt, soll in diesem Kontext nicht verschwiegen werden. s. Müller (Anm. 62) 309 Anm. 53. 335. Für den Hinweis danke ich Herrn I. Weiler.

${ }^{69}$ Zur Thematik der Wechselwirkungen zwischen griechischer und römischer Kultur vgl. zuletzt die Beiträge in E. N. Ostenfeld (Hrsg.), Greek Romans and Roman Greeks. Studies in Cultural Interaction, Aarhus Studies in Mediterranean Antiquity 3 (2002) sowie G. Vogt-Spira - B. Rommel (Hrsg.), Rezeption und Identität. Die kulturelle Auseinandersetzung Roms mit Griechenland als europäisches Paradigma (1999).

70 Gal. protr. 9. 11. - s. dazu Müller (Anm. 62) 296 ff.

71 s. dazu vor allem W. Weismann, Kirche und Schauspiele. Die Schauspiele im Urteil der lateinischen Kirchenväter unter besonderer Berücksichtigung von Augustin, Cassiciacum 27 (1972) bes. 62 ff. 81 ff. mit zahlreichen Quellenangaben.

72 Tert. spect. 18, 2 f.: ... palaestrica diaboli negotium est.

73 Novatianus, De spectaculis 8, 1 f.: ... est manifesta otiosis hominibus negotiatio. 
Konkurrenz zu den sehr beliebten Gladiatorenspielen und Wagenrennen standen ${ }^{74}$. Nicht nur der Agon, sondern auch der Übungsbetrieb, die beide den christlichen Wertvorstellungen nicht entsprachen, waren dieser Kritik ausgesetz ${ }^{75}$. Die tatsächliche Ursache für den kontinuierlichen Rückgang von Agonen und den Niedergang des Sports liegt somit neben ökonomischen Überlegungen hauptsächlich in der Körperfeindlichkeit des Christentums begründet ${ }^{76}$. Daß seit dem 3. Jahrhundert $n$. Chr. immer weniger Bürger bereit und in der Lage waren, die kostspieligen Liturgien wie beispielsweise die Gymnasiarchie zu übernehmen, beschleunigte diesen Prozeß lediglich. Ein voller Betrieb der Gymnasien war zu diesem Zeitpunkt in den meisten Fällen nicht mehr gewährleistet ${ }^{77}$.

Man kann davon ausgehen, daß die Palästren des Hafen-, Theater-, Ost- und Vediusgymnasiums, deren multifunktionale Planung nicht ausgeschlossen werden soll, schon mit der Errichtung dieser Anlagen und besonders nach ihren Umgestaltungen, wie vor allem für das Ostgymnasium und indirekt durch die baulichen Veränderungen in der basilica thermarum des Vediusgymnasium gezeigt werden konnte, vorrangig als Bildungs- und Ausbildungszentren genutzt wurden und somit einen wesentlichen Bestandteil kulturellen und geistigen Lebens in der Stadt bildeten. $\mathrm{Da} ß$ die räumlichen, topographischen und kulturhistorischen Voraussetzungen jegliche sportliche Aktivität apodiktisch ausschließen, soll damit nicht impliziert werden.

\author{
Dr. Martin Steskal \\ Österreichisches Archäologisches Institut, Franz Klein-Gasse 1, A-1190 Wien \\ E-Mail:martin.steskal@oeai.at
}

Abbildungsnachweis: Abb. 1: entnommen dem digitalen Stadtplan von Ephesos (St. Klotz, Ch. Schirmer, ÖAI 1998); Abb. 2. 3. 5: Photo A. Schiffleitner, ÖAI NegNr. 82/95, 30; 68/95, 31; 96/94, 11; Abb. 4. 6 : Photo G. A. Plattner; Abb. 7. 8: ÖAI Wien, Photo N. Gail.

74 Vgl. Weismann (Anm. 71) 63. 83.

75 Vgl. Tert., De pallio 4, 1: ... lutea unctio et pulverea volutatio ... - Auch die Unmäßigkeit der Wettkämpfer kommt in diesem Kontext zur Sprache: Sen. epist. 88, 19: ... vomitores, quorum corpora in sagina, animi in macie et veterno sunt.; Novatianus, De spectaculis 8, 1: In iniuriam suam pinguescit in sagina corpus ut robustius aut feriat aut vapulet, et prima victoria est ultra modum, humani ventris esurire potuisse, super titulo coronatae edacitatis flagitiosae nundinae: ictibus infelix facies locatur, ut infelicior venter saginetur; Tert., De spectaculis 18, 2: ... nusquam tibi ... placebunt ... cura facticii corporis, ut plasticam Dei supergressa, et propter Graeciae otium altiles homines oderis. - Zur grundsätzlichen Kritik am Badebetrieb durch Christen s. F. Yegül, Olbia 8, 2003, bes. $56 \mathrm{ff}$.

76 s. Müller (Anm. 62) $336 \mathrm{ff}$.

77 Vgl. Roueché (Anm. 9) 137: »As was said above, the function of the gymnasia had been to educate the ruling classes of the cities in a manner appropriate to citizens; but from the third century, the purpose of education was increasingly to produce, not the ruling élite of the cities, but the governing class of the empire. For that, the expensive installations of the gymnasium, intended to provide physical as well as intellectual education, were not necessary. What survived was the intellectual, and less expensive, part of that education.« 
